\title{
Strategies for Geriatric Pneumonia in Healthcare Facilities - How Effective is Combined Influenza and Pneumococcal Vaccination?
}

This article was published in the following Dove Press journal:

International Journal of General Medicine

\section{Masafumi Seki (1D \\ Department of Infectious Diseases, Faculty of Medicine, Tohoku Medical and Pharmaceutical University, Sendai City, Japan}

Correspondence: Masafumi Seki Department of Infectious Diseases, Faculty of Medicine, Tohoku Medical and Pharmaceutical University, I-I5-I

Fukumuro, Miyagino-Ku, Sendai City,

Miyagi 983-86/2, Japan

Tel +8I-22-259-|22I

Fax +8I-22-290-8956

Email m-seki@tohoku-mpu.ac.jp

\begin{abstract}
Vaccination is an important strategy to prevent influenza and its related pneumococcal pneumonia. Combined influenza and pneumococcal vaccination should be recommended because of the synergic effects of the two vaccines, compared with either influenza vaccine or pneumococcal vaccine alone.
\end{abstract}

Keywords: influenza virus, Streptococcus pneumoniae, nursing and healthcare-associated pneumonia, NHCAP, mortality, cost-effectiveness

\section{Introduction}

The pneumococcal vaccination rate in Japan remains below 20\%, lagging far behind the $70 \%$ rate in the USA; however, this rate has increased in recent years, reaching about $30 \%$ in 2019 , and is expected to increase further in the future. ${ }^{1}$

Pneumococcal pneumonia following influenza is an important issue; however, influenza vaccine and pneumococcal vaccine are both effective in preventing severe pneumonia due to influenza and Streptococcus pneumoniae coinfection, and may modulate the innate and acquired immunity to $S$. pneumoniae and influenza virus. ${ }^{2,3}$ Chemokine storms, such as higher MCP-1 levels and lower levels of CXCL1, IFN$\gamma$, IL-17A, and IL-10, were detected in pneumococcal conjugate vaccination (PCV)treated influenza-related pneumococcal mice, and improved the survival of these mice. $^{2}$ The protective effect of influenza immunization against bacterial infections has also been suggested. ${ }^{3}$ Therefore, synergic effects of simultaneous vaccination could be expected clinically.

With growing evidence for the benefits of combined vaccination with the influenza and pneumococcal vaccines, particularly in the elderly, the time for simultaneous influenza and pneumococcal vaccination has arrived in Japan.

\section{Evidence on Combined Use of Both Vaccines}

Secondary pneumococcal pneumonia is known to be a common and serious complication of influenza, and we have conclusively demonstrated this using both clinical data from humans and animal experiments. ${ }^{4,5}$ Pneumococcal pneumonia patients with influenza virus infection showed higher severity and inflammation than patients with either pneumococcal pneumonia alone or influenza infection alone. ${ }^{5}$ Extreme severe pneumonia with lung hemorrhage was found frequently in mice coinfected with influenza virus and $S$. pneumoniae, whereas only mild to 
moderate pneumonia was found when they were infected with influenza virus alone or S. pneumoniae alone. ${ }^{4}$

Furthermore, in a similar influenza-related secondary bacterial pneumonia mouse model, McCullers showed that treatment with anti-influenza agents alone significantly reduced the mortality compared with treatment with the antibiotics without anti-influenza agents. ${ }^{6}$ These data suggested that anti-influenza drugs are more effective than antibiotics, and demonstrate the benefit of more advanced and earlier care for the virus, including vaccines and infection-control strategies, compared with treatment with antimicrobial agents. ${ }^{4,5}$ Therefore, vaccination may be a far more important strategy than antimicrobial treatment.

One of the earliest studies of combination vaccination was by Swedish researchers Christenson et al, who investigated the effects of vaccination with both pneumococcal and influenza vaccines. $^{7}$ About 260,000 Stockholm residents $\geq 65$ years old were invited by mail to receive both vaccinations, ie, trivalent, split-virion, influenza vaccines and the 23-valent, pneumococcal polysaccharide vaccines, and at the end of the vaccination campaign, $76 \%$ of the 100,000 residents had been vaccinated with both. When the researchers compared the effect of vaccination between these groups and the unvaccinated group (about 160,000 residents), they found that the group given both vaccines showed significantly lower rates of hospitalization for pneumonia (29\% decrease) and pneumococcal pneumonia ( $36 \%$ decrease). This may be due to lower rates of both influenza with and without pneumonia patients were decreased ( $46 \%$ decrease). In addition, a significantly lower total mortality ( $57 \%$ decrease) was found in this study, according to the decrease in hospitalization for invasive pneumococcal disease (52\% decrease).

In Japan, a randomized, double-blinded, placebo-controlled study of 23-valent pneumococcal vaccine was conducted in 2010 among elderly care-home residents. ${ }^{8}$ Among the 1006 residents (mean age 84.7 years) at several elderly care facilities, a group vaccinated with 23 -valent pneumococcal vaccine (502 participants) and a placebo group given an intramuscular injection of physiological saline (504 participants) were followed up for around 3 years. The primary endpoints were onset of pneumonia and onset of pneumococcal pneumonia, while secondary endpoints were death due to pneumonia and death due to pneumococcal pneumonia. Although detailed information on the influenza vaccine in these patients was lacking, the 3 -year follow-up found that pneumonia occurred in the vaccinated group at a rate of 55 cases per 1000 personyears, with 12 cases of pneumococcal pneumonia per 1000 person-years. In the placebo group, pneumonia occurred at a rate of 91 cases per 1000 person-years, with 32 cases of pneumococcal pneumonia per 1000 person-years. Consistent with the Swedish study, this Japanese study demonstrated that vaccination, including pneumococcal vaccine alone, curtailed significantly the onset of pneumococcal pneumonia, by $63.8 \%$, and the onset of all pneumonia, by $44.8 \%$, in the elderly. More significant reduction of the total pneumonia rate, including influenza-related pneumonia, may be shown when simultaneous vaccination against both influenza virus and $S$. pneumoniae is performed.

Furthermore, looking at death as the secondary endpoint, mortality rates from all pneumonia were $25.0 \%$ (26/ $104)$ in the placebo group and $20.6 \%(13 / 63)$ in the vaccinated group, showing no significant difference. However, in patients who contracted pneumococcal pneumonia, this disease was the cause of death in 13 of 37 participants $(35.1 \%)$ in the placebo group, but no participants in the vaccinated group. This finding demonstrates that vaccination can significantly suppress death due to pneumococcal pneumonia according to the decreased onset of pneumonia as the primary endpoint of this study, although this favorable result may be due to the reduction of invasive pneumococcal disease resulting from pneumonia.

Another study demonstrated that overall medical costs due to geriatric pneumonia in Japan were reduced by administering the pneumococcal vaccine together with the influenza vaccine. ${ }^{9}$ About 800 elderly participants ( $\geq 65$ years old) were divided into a group receiving both the trivalent, splitvirion, influenza vaccine and the 23 -valent, pneumococcal polysaccharide pneumococcal vaccine (combined vaccine group, 394 participants) and a group receiving only the trivalent, split-virion, influenza vaccine (single vaccine group, 392 participants). Comparison of pneumonia incidence, number of pneumonia episodes, number of hospitalizations, and total medical costs during the 2 years after vaccination revealed that among those $\geq 75$ years old, rates of hospitalization for pneumonia were $41.5 \%$ lower in the combined vaccine group $(P=0.039)$. Among elderly participants with difficulty walking, frequency of hospitalization for pneumonia in the 2 years after vaccination was significantly decreased, by $62.7 \%$, in the combined vaccine group $(P=0.005)$. In detail, total pneumonia and respiratory infection-related medical costs, such as outpatient and/or inpatient medication fees in the combined vaccine group at 1 year after vaccination, were around $¥ 57,000$, representing a saving of around $¥ 80,000$ compared to the total medical costs of 
around $¥ 140,000$ in the single vaccine group $(P=0.027)$. These results suggest that both influenza and pneumococcal vaccines may have more benefit not only clinically, but also cost-effectively, than the pneumococcal vaccination alone. Expanding the provision of pneumococcal vaccination would appear crucial to cutting medical costs. In fact, the 23 -valent vaccine, in particular, has been calculated to be one of the most highly cost-effective vaccines.

Publicly assisted vaccinations against influenza and pneumococcal diseases for elderly people are available in a number of countries, including the USA and various European nations. In Japan, among the series of pneumonia guidelines issued by the Japanese Respiratory Society, the 2011 Nursing- and Healthcare-Associated Pneumonia (NHCAP) guideline was the first to emphasize the importance of vaccination against influenza and $S$. pneumoniae for elderly patients to prevent influenza-related pneumococcal pneumonia, one of the representative high-age pneumonia types in Japan. ${ }^{10}$ Thus, since 2014, Japan has provided public assistance for pneumococcal vaccination for elderly people across the country. ${ }^{1}$

A 2018 study demonstrated the safety and immunogenicity of revaccination with 23-valent pneumococcal polysaccharide vaccine at intervals of more than 5 years in elderly Japanese people. ${ }^{11}$ Moreover, the combination of a new quadrivalent influenza vaccine and 23-valent pneumococcal polysaccharide vaccine was suggested to have an acceptable immunogenicity that is comparable to sequential administration without an increase in adverse reactions for elderly Japanese people. ${ }^{12}$

We have two types of pneumococcal vaccine, ie, 13valent conjugate vaccine and 23 -valent polysaccharide vaccine. The conjugate vaccine can induce strong immunogenicity as a booster, and the polysaccharide vaccine covers the wide range of serotypes of $S$. pneumoniae; therefore, it may be better for both types of pneumococcal vaccine to be received in sequence for elderly people. ${ }^{13,14}$

Vaccination rates for pneumococcus will be increased in due course with the combination of influenza vaccine in Japan.

\section{Conclusion}

The growing understanding of the importance of pneumococcal vaccination for the elderly should lead to more widespread uptake, and combined use with the influenza vaccine is expected to increase the effectiveness of these vaccines in Japan.

\section{Disclosure}

The author reports no conflicts of interest for this work.

\section{References}

1. Suzuki M, Dhoubhadel BG, Ishifuji T, et al. Adult Pneumonia Study Group-Japan (APSG-J). Serotype-specific effectiveness of 23-valent pneumococcal polysaccharide vaccine against pneumococcal pneumonia in adults aged 65 years or older: a multicentre, prospective, test-negative design study. Lancet Infect Dis. 2017;17:313-321. doi:10.1016/S1473-3099(17)30049-X

2. Mimura K, Kimura S, Kajiwara C, et al. Pneumococcal conjugate vaccine modulates macrophage-mediated innate immunity in pneumonia caused by Streptococcus pneumoniae following influenza. Microbes Infect. 2020.

3. Christopoulou I, Roose K, Ibañez LI, Saelens X. Influenza vaccines to control influenza-associated bacterial infection: where do we stand? Expert Rev Vaccines. 2015;14:55-67. doi:10.1586/ 14760584.2015.957191

4. Seki M, Yanagihara K, Higashiyama Y, et al. Immunokinetics in severe pneumonia due to influenza virus and bacteria coinfection in mice. Eur Respir J. 2004;24:143-149. doi:10.1183/ 09031936.04.00126103

5. Seki M, Kosai K, Yanagihara K, et al. Disease severity in patients with simultaneous influenza and bacterial pneumonia. Intern Med. 2007;46:953-958. doi:10.2169/internalmedicine.46.6364

6. McCullers JA. Effect of antiviral treatment on the outcome of secondary bacterial pneumonia after influenza. $J$ Infect Dis. 2004;190:519-526. doi:10.1086/421525

7. Christenson B, Lundbergh P, Hedlund J, Ortqvist A. Effects of a large-scale intervention with influenza and 23 -valent pneumococcal vaccines in adults aged 65 years or older: a prospective study. Lancet. 2001;357:1008-1011. doi:10.1016/S0140-6736(00)04237-9

8. Maruyama T, Taguchi O, Niederman MS, et al. Efficacy of 23-valent pneumococcal vaccine in preventing pneumonia and improving survival in nursing home residents: double blind, randomised and placebo controlled trial. BMJ. 2010;340:c1004-c1004. doi:10.1136/bmj. c1004

9. Kawakami K, Ohkusa Y, Kuroki R, et al. Effectiveness of pneumococcal polysaccharide vaccine against pneumonia and cost analysis for the elderly who receive seasonal influenza vaccine in Japan. Vaccine. 2010;28:7063-7069. doi:10.1016/j.vaccine.2010.08.010

10. Kohno S, Imamura Y, Shindo Y, et al. Clinical practice guidelines for nursing- and healthcare-associated pneumonia (NHCAP). Respir Investig. 2013;51:103-126. doi:10.1016/j.resinv.2012.11.001

11. Kawakami K, Kishino H, Kanazu S, et al. Time interval of revaccination with 23 -valent pneumococcal polysaccharide vaccine more than 5 years does not affect the immunogenicity and safety in the Japanese elderly. Hum Vaccin Immunother. 2018;14:1931-1938. doi:10.1080/21645515.2018.1456611

12. Nakashima K, Aoshima M, Ohfuji S, et al. Immunogenicity of simultaneous versus sequential administration of a 23 -valent pneumococcal polysaccharide vaccine and a quadrivalent influenza vaccine in older individuals: a randomized, open-label, non-inferiority trial. Hum Vaccin Immunother. 2018;14:1923-1930. doi:10.1080/ 21645515.2018.1455476

13. Clutterbuck EA, Lazarus R, Yu LM, et al. Pneumococcal conjugate and plain polysaccharide vaccines have divergent effects on antigenspecific B cells. J Infect Dis. 2012;205:1408-1416. doi:10.1093/ infdis/jis 212

14. Tomczyk S, Bennett NM, Stoecker C, et al. Use of 13-valent pneumococcal conjugate vaccine and 23 -valent pneumococcal polysaccharide vaccine among adults aged $\geq 65$ years: recommendations of the Advisory Committee on Immunization Practices (ACIP). MMWR. 2014;63:822-825. 


\section{Publish your work in this journal}

The International Journal of General Medicine is an international, peer-reviewed open-access journal that focuses on general and internal medicine, pathogenesis, epidemiology, diagnosis, monitoring and treatment protocols. The journal is characterized by the rapid reporting of reviews, original research and clinical studies across all disease areas. The manuscript management system is completely online and includes a very quick and fair peer-review system, which is all easy to use. Visit http://www.dovepress.com/ testimonials.php to read real quotes from published authors. 